\title{
Proceedings of the Thirteenth Annual UT- KBRIN Bioinformatics Summit 2014
}

\author{
Eric C. Rouchka ${ }^{1 *}$, Julia H. Chariker ${ }^{2}$ \\ From UT-KBRIN Bioinformatics Summit 2014 \\ Cadiz, KY, USA. 11-13 April 2014
}

The University of Tennessee (UT) and the Kentucky Biomedical Research Infrastructure Network (KBRIN) have collaborated over the past thirteen years to share research and educational expertise in bioinformatics. One result is an annual regional summit for researchers, educators and students interested in bioinformatics. The Thirteenth Annual UT- KBRIN Bioinformatics Summit was held at Lake Barkley State Park in Cadiz, Kentucky on April 1113, 2014. A total of 172 participants pre-registered, with 104 from Tennessee and 61 from Kentucky. Among the registrants were 69 faculty, 45 students, 38 staff, and 20 postdocs. The conference program consisted of a workshop on Cytoscape and three days of presentations broken into plenary sessions on Genetic Variation and Mutational Analysis, Genomics, and P4 Systems Biology. Nine short talks were selected from 51 submitted poster abstracts.

\section{Friday workshops}

Keiichiro Ono (UCSD) opened the Summit with the workshop "PART I: Introduction to Cytoscape: an Open Source Platform for Biological Network Data Analysis and Visualization." He provided a description of the basic UI, methods for importing and exporting data, and methods for highlighting and exploring information in a biological network [1-3]. Keiichiro discussed methods for importing publicly available databases with a network structure, such as STRING [4], IntAct [5], BioGRID [6], ChEMBL [7], KEGG [8], Reactome [9], WikiPathways [10], and PathwayCommons [11].

Keiichiro presented a number of commands for simplifying networks, including selecting first neighbors of a node, creation of a subgraph, and filtering based on node and edge attributes. He also demonstrated tools for highlighting

\footnotetext{
* Correspondence: eric.rouchka@louisville.edu

1Department of Computer Engineering and Computer Science, University of Louisville, Duthie Center for Engineering, Louisville, KY 40292, USA Full list of author information is available at the end of the article
}

information in a network, including changing the visual properties of nodes and edges.

The second workshop, "PART II: Hands-On: Biological Network Analysis and Visualization with Cytoscape," focused on advanced topics, including effective visualization techniques and external network analysis. Keiichiro provided a description of the network layouts available, such as hierarchical, force-directed, circular, and manual. In addition, he provided guidelines for choosing the most effective visual and spatial properties.

\section{Session I: genetic variation and mutational analysis}

Hannah Carter (UCSD) began the formal program with "Identifying cancer drivers from high-throughput sequencing data" which focused on single nucleotide somatic mutations occurring in cancer samples. The goal was to determine which mutations are "drivers" contributing to tumorogenesis by altering protein activity, and which are "passengers" in the process. This is complicated since genomic abnormalities from 50 cancer types shows only a small number of mutations drive tumor progression [12]. She closed by describing the Cancer-Specific HighThroughput Annotation of Somatic Mutations (CHASM) approach for prioritizing driver SNVs [13].

Travis Burleson (Affymetrix) concluded the Friday evening session with "Mapping changes in the transcriptome: A primer into alternative splicing and how the Affymetrix TAC 2.0 software estimates these events." Affymetrix has recently developed an array, HTAv2, for analysing alternative splicing in humans. Travis discussed in detail both the chip platform as well as the Transcript Analysis Console (TAC).

Saturday morning began with the final presentation in Genetic Variation and Mutational Analysis. Steve Horvath (UCLA) presented "Empirical evaluation of predictionand correlation network methods applied to genomic 
data." The bulk of the presentation focused on weighted gene co-expression network analysis (WGCNA) for identifying clusters or modules of highly correlated genes [14]. One of the key aspects of WGCNA is that it identifies a single gene within each module, called an eigengene, which best represents the pattern of gene expression found across all genes in the module. Dr. Horvath pointed out that identification of intramodular hub genes provides more meaningful information about a biological network than identification of hubs within the entire network. He also discussed a prediction method called the random generalized linear model (RGLM) [15] which is a combination of a random forest $[16,17]$ and a forward regression model.

\section{Session II: genomics}

Dr. Alistair Forrest (RIKEN Center for Life Science Technologies) opened the Genomics session on Saturday morning with "FANTOM5 - a mammalian promoter level analysis." The FANTOM (Functional ANnoTation Of the Mammalian genome) project is an international research consortium established in 2000 with an initial goal of annotating over 20,000 cDNAs sequenced as part of the RIKEN Mouse Gene Encyclopedia Project [18]. The aim of FANTOM 5 was to generate a map of most human promoters as well as to generate comparative transcriptional network models of each cellular state.

Dr. Forrest focused on the results presented in three manuscripts published just prior to the Summit. The first, "A promoter level mammalian expression atlas" [19] reveals the results of CAGE across 975 human and 399 mouse samples, including primary cells and cancer cell lines. The second, "An atlas of active enhancers across human cell types and tissues" [20], identified 43,011 enhancer candidates within 432 primary cell samples, 135 tissue samples, and 241 cell line samples, all from humans, using overlaid ChIP-seq and CAGE sequencing results within transcription start sites. The third paper, "Interactive Visualization and analysis of large-scale NGS data-sets using ZENBU," focused on the visualization toolkit ZENBU created for analysing large-scale sequencing datasets [21].

John Zhang (Life Technologies) closed the genomics session on Saturday evening with a discussion of the Ion Torrent PGM ${ }^{\mathrm{TM}}$ and Proton ${ }^{\mathrm{TM}}$ instruments. John discussed a number of applications available on these machines, with a specific focus on the Ion AmpliSeq ${ }^{\text {TM }}$ panels for detecting single nucleotide variants (SNVs) and insertions and deletions (indels). John focused on demonstrating the Ion Reporter ${ }^{\text {TM }}$ software for bioinformatics analysis [22].

\section{Session III: P4 systems biology}

Nathan Price (The Institute for Systems Biology) kicked off the P4 Systems Biology session on Sunday morning with "Harnessing omics data for biological and medical discovery." Dr. Price discussed several projects focused on systems approaches to integrating omics data in order to approach medicine from a personalized perspective. Included in his discussion was a project looking at honey bees as a model organism for examining social behaviors such as aggression, maturation, and foraging [23]. Dr. Price also presented a project that illustrates the usefulness of the Allen Brain Atlas [24] for characterization of cell type-specific genes [25] in which it was discovered that positional clustering of 170 neuron-specific genes reproduced the brain's spatial structure. He also described SNAPR, a pipeline for RNA-seq alignment and analysis [26]. SNAPR contains a very efficient alignment algorithm, running approximately 25 times faster than TopHat [27] and Bowtie [28]. The final topic focused on P4 (predictive, preventative, personalized, and participatory) medicine [29]. He described in detail two projects currently underway at The Institute for Systems Biology - the Pioneer 100 project and the ISB 100K Wellness Project [30,31].

Joel Dudley (Icahn School of Medicine at Mount Sinai) concluded the invited speaker portion of the summit with "Integrating the digital universe of information for better models of disease and drug response." Dr. Dudley's presentation focused on medical discovery through big data bioinformatics. He described the BioMe Biobank project at Mount Sinai, which combines big data and personalized medicine with the goal of collecting samples from 100,000 donors. Dr. Dudley emphasized the importance of using publicly available data for a variety of research questions. In addition, he discussed the development of immune-pharmacology networks based on a variety of high-dimensional data [32].

\section{Posters and short talks}

The poster session was held on day two. Fifty-one posters were on display from a variety of different research areas. A number of posters were also selected for short talks. These included "A new set-valued system identification approach to identifying rare genetic variants for ordered categorical phenotype" (Guolian Kang, St. Jude Children's Research Hospital); "Building a knowledge base to assist clinical decision-making using the Pediatric Research Database (PRD) and machine learning: A case study on pediatric asthma patients" (Naga Nagisetty, Le Bonheur Children's Hospital); "Differential isoform expression provides comprehensive stage-dependent signatures in cancer" (Qi Liu, Vanderbilt University); "Piecing the puzzle together: a revisit to transcript reconstruction problem in RNA-seq" (Yan Huang, University of Kentucky); "An island-based approach for differential expression analysis" (Abdallah Eteleeb, University of Louisville); "Evaluating four major algorithms for identifying differential regulators 
in condition-specific transcriptional responses" (Hui Yu, Vanderbilt University); "Development of sparse Bayesian multinomial generalized linear model for multi-class prediction" (Behrouz Madahian, University of Memphis); "Transcriptome profile of OVCAR3 cisplatin-resistant ovarian cancer cell line" (Sammed Madape, Meharry Medical College); and "Development of large-scale metabolite identification methods for metabolomics" (Hunter Moseley, University of Kentucky). For full author lists and abstracts see the rest of the supplement.

\section{Future plans}

The 2015 Bioinformatics summit will return to Tennessee in the spring of 2015. Potential areas include current trends in molecular biology, applications of next-generation sequencing, and systems biology.

\section{Competing interests}

The authors declare that they have no competing interests.

\section{Authors' contributions}

ECR served on the program committee for the UT-KBRIN Bioinformatics Summit and collected the final abstracts from selected authors. ECR and JHC contributed equally to the writing of the meeting summary.

\section{Acknowledgements}

We would like to thank the Conference Program Committee members Nigel Cooper (University of Louisville), Dan Goldowitz (University of British Columbia), Mike Langston (University of Tennessee-Knoxville), Terry MarkMajor (University of Tennessee-Memphis), Claire Rinehart (Western Kentucky University), Arnold Stromberg (University of Kentucky), Rob Williams (University of Tennessee-Memphis), and Zhongming Zhao (Vanderbilt University) for organizing an outstanding scientific program. In addition, we wish to thank Terry Mark-Major, Michelle Padgett, Whitney Rogers, and Susan Boucher for their efforts in handling conference organization details. Funding for the UT- KBRIN Summit is provided in part by the University of Memphis Office of the Provost, Memphis Research Consortium, Kentucky Biomedical Research Infrastructure Network (KBRIN), University of Tennessee Center for Integrative and Translational Genomics, University of Tennessee Molecular Resource Center, and NIH grant P20GM103436.

\section{Declarations}

Publication of this supplement was funded, in part, by the National Institutes of Health $(\mathrm{NIH})$ and the National Institute of General Medical Sciences (NIGMS) under grant P20GM103436. The article contents are solely the responsibility of the authors and do not represent the official views of $\mathrm{NIH}$ or NIGMS.

\section{Authors' details}

'Department of Computer Engineering and Computer Science, University of Louisville, Duthie Center for Engineering, Louisville, KY 40292, USA. ${ }^{2}$ Department of Psychological and Brain Sciences, University of Louisville, Louisville, KY 40292, USA.

\section{Published: 26 September 2014}

\section{References}

1. Cline MS, Smoot M, Cerami E, Kuchinsky A, Landys N, Workman C, Christmas R, Avila-Campilo I, Creech M, Gross B, et al: Integration of biological networks and gene expression data using Cytoscape. Nature protocols 2007, 2(10):2366-2382.

2. Shannon P, Markiel A, Ozier O, Baliga NS, Wang JT, Ramage D, Amin N, Schwikowski B, Ideker T: Cytoscape: a software environment for integrated models of biomolecular interaction networks. Genome research 2003, 13(11):2498-2504.
3. Smoot ME, Ono K, Ruscheinski J, Wang PL, Ideker T: Cytoscape 2.8: new features for data integration and network visualization. Bioinformatics 2011, 27(3):431-432.

4. Jensen $L$, Kuhn M, Stark M, Chaffron S, Creevey C, Muller J, Doerks T, Julien P, Roth A, Simonovic M, et al: STRING 8-a global view on proteins and their functional interactions in 630 organisms. Nucleic acids research 2009, 37(Database issue):D412-416.

5. Orchard S, Ammari M, Aranda B, Breuza L, Briganti L, Broackes-Carter F, Campbell NH, Chavali G, Chen C, del-Toro N, et al: The MIntAct projectIntAct as a common curation platform for 11 molecular interaction databases. Nucleic acids research 2014, 42(Database issue):D358-363.

6. Chatr-Aryamontri A, Breitkreutz BJ, Heinicke S, Boucher L, Winter A, Stark C, Nixon J, Ramage L, Kolas N, O'Donnell L, et al: The BioGRID interaction database: 2013 update. Nucleic acids research 2013, 41(Database issue) D816-823.

7. Bento AP, Gaulton A, Hersey A, Bellis $\sqcup$, Chambers J, Davies M, Kruger FA, Light Y, Mak L, McGlinchey S, et al: The ChEMBL bioactivity database: an update. Nucleic acids research 2014, 42(Database issue):D1083-1090.

8. Kanehisa M, Goto S: KEGG: kyoto encyclopedia of genes and genomes. Nucleic acids research 2000, 28(1):27-30.

9. Croft D, Mundo AF, Haw R, Milacic M, Weiser J, Wu G, Caudy M, Garapati P, Gillespie M, Kamdar MR, et al: The Reactome pathway knowledgebase. Nucleic acids research 2014, 42(Database issue):D472-477.

10. Kelder T, van lersel MP, Hanspers K, Kutmon M, Conklin BR, Evelo CT, Pico AR: WikiPathways: building research communities on biological pathways. Nucleic acids research 2012, 40(Database issue):D1301-1307.

11. Cerami EG, Gross BE, Demir E, Rodchenkov I, Babur O, Anwar N, Schultz N, Bader GD, Sander C: Pathway Commons, a web resource for biological pathway data. Nucleic acids research 2011, 39(Database issue):D685-690.

12. Gonzalez-Perez A, Mustonen V, Reva B, Ritchie GR, Creixell P, Karchin R, Vazquez M, Fink JL, Kassahn KS, Pearson JV, et al: Computational approaches to identify functional genetic variants in cancer genomes. Nature methods 2013, 10(8):723-729.

13. Wong WC, Kim D, Carter H, Diekhans M, Ryan MC, Karchin R: CHASM and SNVBox: toolkit for detecting biologically important single nucleotide mutations in cancer. Bioinformatics 2011, 27(15):2147-2148.

14. Langfelder $P$, Horvath S: WGCNA: an R package for weighted correlation network analysis. BMC bioinformatics 2008, 9:559.

15. Song L, Langfelder P, Horvath S: Random generalized linear model: a highly accurate and interpretable ensemble predictor. BMC bioinformatics 2013, 14:5.

16. Breiman L: Random forests. Machine learning 2001, 45(1):5-32.

17. Cutler A, Zhao G: PERT-perfect random tree ensembles. Computing Science and Statistics 2001, 33:490-497.

18. Kawai J, Shinagawa A, Shibata K, Yoshino M, Itoh M, Ishii Y, Arakawa T, Hara A, Fukunishi $Y$, Konno $H$, et al: Functional annotation of a full-length mouse cDNA collection. Nature 2001, 409(6821):685-690.

19. The Fantom Consortium: A promoter-level mammalian expression atlas. Nature 2014, 507(7493):462-470.

20. Andersson R, Gebhard C, Miguel-Escalada I, Hoof I, Bornholdt J, Boyd M, Chen Y, Zhao X, Schmidl C, Suzuki T, et al: An atlas of active enhancers across human cell types and tissues. Nature 2014, 507(7493):455-461.

21. Severin J, Lizio M, Harshbarger J, Kawaji H, Daub CO, Hayashizaki Y, Bertin N, Forrest AR: Interactive visualization and analysis of large-scale sequencing datasets using ZENBU. Nature biotechnology 2014, 32(3):217-219.

22. Ion Reporter | Life Technologies [https://ionreporter.lifetechnologies. com/ir/].

23. Chandrasekaran S, Ament SA, Eddy JA, Rodriguez-Zas SL, Schatz BR, Price ND, Robinson GE: Behavior-specific changes in transcriptional modules lead to distinct and predictable neurogenomic states. Proceedings of the National Academy of Sciences of the United States of America 2011, 108(44):18020-18025.

24. Lein ES, Hawrylycz MJ, Ao N, Ayres M, Bensinger A, Bernard A, Boe AF, Boguski MS, Brockway KS, Byrnes EJ, et al: Genome-wide atlas of gene expression in the adult mouse brain. Nature 2007, 445(7124):168-176.

25. Ko Y, Ament SA, Eddy JA, Caballero J, Earls JC, Hood L, Price ND: Cell typespecific genes show striking and distinct patterns of spatial expression in the mouse brain. Proceedings of the National Academy of Sciences of the United States of America 2013, 110(8):3095-3100.

26. SNAPR: a bioinformatics pipeline for efficient and accurate RNA-seq alignment and analysis [http://price.systemsbiology.net/SNAPR] . 
27. Kim D, Pertea G, Trapnell C, Pimentel H, Kelley R, Salzberg SL: TopHat2: accurate alignment of transcriptomes in the presence of insertions, deletions and gene fusions. Genome biology 2013, 14(4):R36.

28. Langmead B, Trapnell C, Pop M, Salzberg SL: Ultrafast and memoryefficient alignment of short DNA sequences to the human genome. Genome biology 2009, 10(3):R25.

29. Auffray C, Charron D, Hood L: Predictive, preventive, personalized and participatory medicine: back to the future. Genome medicine 2010, 2(8):57.

30. Gibbs WW: Medicine gets up close and personal. Nature 2014, 506(7487):144-145

31. 100K Wellness project [http://research.systemsbiology.net/100k/].

32. Kidd BA, Peters LA, Schadt EE, Dudley JT: Unifying immunology with informatics and multiscale biology. Nature immunology 2014, 15(2):118-127.

doi:10.1186/1471-2105-15-S10-11

Cite this article as: Rouchka and Chariker: Proceedings of the Thirteenth Annual UT- KBRIN Bioinformatics Summit 2014. BMC Bioinformatics 2014 15(Suppl 10):11.

\section{Submit your next manuscript to BioMed Central} and take full advantage of:

- Convenient online submission

- Thorough peer review

- No space constraints or color figure charges

- Immediate publication on acceptance

- Inclusion in PubMed, CAS, Scopus and Google Scholar

- Research which is freely available for redistribution

Submit your manuscript at www.biomedcentral.com/submit
Ciomed Central 